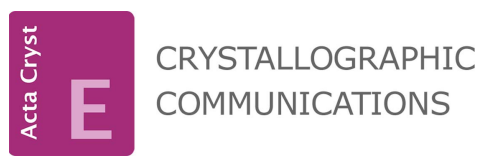

ISSN 2056-9890

Received 9 May 2015

Accepted 19 May 2015

Edited by A. J. Lough, University of Toronto, Canada

Keywords: crystal structure; silver; mixed valency; quinoline

CCDC reference: 1401857

Supporting information: this article has supporting information at journals.iucr.org/e

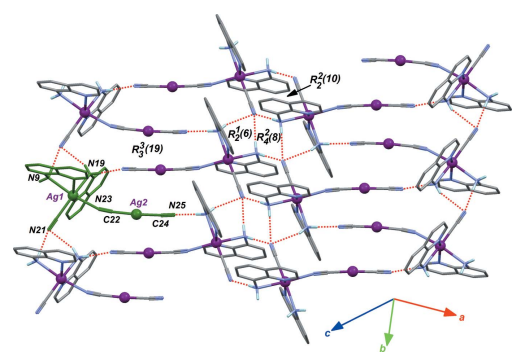

OPEN $\odot$ ACCESS

\section{Crystal structure of $\mu$-cyanido-1:2 $\kappa^{2} \mathrm{~N}: C$-dicyanido- $1 \kappa C, 2 \kappa C$-bis (quinolin-8-amine-1 $\kappa^{2} N, N^{\prime}$ )-2-silver(I)- 1-silver(II): rare occurrence of a mixed-valence $\mathrm{Ag}^{\mathrm{I}, \mathrm{II}}$ compound}

\author{
Zouaoui Setifi, ${ }^{\mathrm{a}, \mathrm{b}}$ Sylvain Bernès, ${ }^{\mathrm{c} *}$ Olivier Pérez, ${ }^{\mathrm{d}}$ Fatima Setifi $^{\mathrm{a} *}$ and \\ Djamil-Azzeddine Rouag ${ }^{b}$
}

a Laboratoire de Chimie, Ingénierie Moléculaire et Nanostructures (LCIMN), Université Ferhat Abbas Sétif 1, Sétif 19000, Algeria, ' ${ }^{\mathbf{S}}$ nité de Recherche de Chimie de I'Environnement et Moléculaire Structurale (CHEMS), Université Constantine 1, Constantine 25000, Algeria, ' Instituto de Física, Benemérita Universidad Autónoma de Puebla, Av. San Claudio y 18 Sur, 72570 Puebla, Pue., Mexico, and 'Laboratoire CRISMAT, UMR 6508 CNRS, ENSICAEN, 6 Boulevard du Maréchal Juin, 14050 Caen Cedex 04, France. *Correspondence e-mail: sylvain_bernes@Hotmail.com, fat_setifi@yahoo.fr

The title dinuclear complex, $\left[\mathrm{Ag}_{2}(\mathrm{CN})_{3}\left(\mathrm{C}_{9} \mathrm{H}_{8} \mathrm{~N}_{2}\right)_{2}\right]$, may be considered as an $\mathrm{Ag}^{\mathrm{II}}$ compound with the corresponding metal site coordinated by two bidentate quinolin-8-amine molecules, one cyanide group and one dicyanidoargentate(I) anion, $\left[\mathrm{Ag}(\mathrm{CN})_{2}\right]^{-}$. Since this latter ligand contains an $\mathrm{Ag}^{\mathrm{I}}$ atom, the complex should be a class 1 or class 2 mixed-valence compound, according to the RobinDay classification. The $\mathrm{Ag}^{\mathrm{II}}$ atom is six-coordinated in a highly distorted octahedral geometry, while the $\mathrm{Ag}^{\mathrm{I}}$ atom displays the expected linear geometry. In the crystal, the amino groups of the quinolin-8-amine ligands form $\mathrm{N}-\mathrm{H} \cdots \mathrm{N}$ hydrogen bonds with the $\mathrm{N}$ atoms of the non-bridging cyanide ligands, forming a two-dimensional network parallel to (102). The terminal cyanide ligands are not engaged in polymeric bonds and the title compound is an authentic molecular complex. The title molecule is thus a rare example of a stable $\mathrm{Ag}^{\mathrm{I}, \mathrm{II}}$ complex, and the first mixed-valence $\mathrm{Ag}^{\mathrm{I}, \mathrm{II}}$ molecular complex characterized by X-ray diffraction.

\section{Chemical context}

The coordination chemistry of silver is clearly dominated by $\mathrm{Ag}^{\mathrm{I}}$ complexes. The oxidation state $\mathrm{Ag}^{\mathrm{II}}$, with a paramagnetic $4 d^{9}$ electronic configuration, is however present in inorganic species like $\mathrm{AgF}_{2}$, a compound which readily decomposes in water, and is even able to oxidize $\mathrm{SiCl}_{4}$ (Grochala \& Mazej, 2015). $\mathrm{Ag}^{\mathrm{II}}$ is also stable in bimetallic perfluorinated compounds $\mathrm{Ag}^{\mathrm{II}} M^{\mathrm{IV}} \mathrm{F}_{6}$, with $M=\mathrm{Pt}, \mathrm{Pd}, \mathrm{Ti}, \mathrm{Rh}, \mathrm{Sn}$ and $\mathrm{Pb}$. In these solids, the $\mathrm{Ag}^{\mathrm{II}}$ sites are bonded to six $\mathrm{F}$ atoms, in an octahedral coordination geometry distorted by the JahnTeller effect. In contrast, $\mathrm{AgO}$, precipitated from $\mathrm{Ag}$ in presence of $\mathrm{K}_{2} \mathrm{~S}_{2} \mathrm{O}_{8}$ in a basic medium, is a diamagnetic mixedvalence $\mathrm{Ag}^{\mathrm{I}, \mathrm{III}}$ oxide, rather than a $\mathrm{Ag}^{\mathrm{II}}$ compound (Housecroft \& Sharpe, 2012). Some actual $\mathrm{Ag}^{\mathrm{II}}$ coordination complexes may be formed in solution, for example $\left[\mathrm{Ag}(\mathrm{bpy})_{2}\right]^{2+}$, which follows the Curie law with a magnetic moment close to the spin-only value expected for a $d^{9}$ system (Kandaiah et al., 2012).

Recently, polynitrile and cyanidometallate anions have received considerable attention because of their importance in both coordination chemistry and in molecular materials chemistry (Atmani et al., 2008; Benmansour et al., 2008, 2009, 2012; Setifi et al., 2013; Setifi, Lehchili et al., 2014; Setifi, Charles et al., 2014). In view of the possible roles of these 
versatile anionic ligands, we have been interested in using them in combination with other chelating or bridging neutral co-ligands to explore their structural and electronic characteristics in the extensive field of molecular materials exhibiting the spin-crossover (SCO) phenomenon (Dupouy et al., 2008, 2009; Setifi et al., 2009; Setifi, Charles et al., 2014; Setifi, Milin et al., 2014). During the course of attempts to prepare such complexes, using the dicyanidoargentate(I) anion, we isolated the title compound, whose structure is described here.

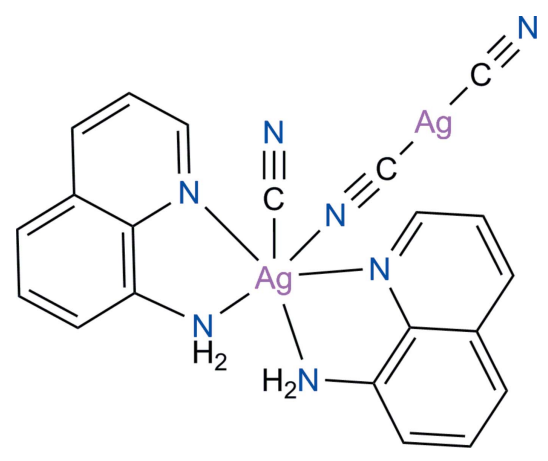

\section{Structural commentary}

The title complex (Fig. 1) is a binuclear silver compound placed in a general position, in which metallic sites present contrasting coordination environments. Ag1 is six-coordinated by two quinolin-8-amine bidentate ligands, one terminal cyanide ligand, and one bridging cyanide ligand. The quinoline ring system $\mathrm{N} 1-\mathrm{C} 8$ is slightly twisted, with a r.m.s. deviation of $0.04 \AA$ A, while the other, N11-C18, may be considered as planar (rms deviation: $0.01 \AA$ ). Quinoline ligands are arranged cis in the octahedral coordination polyhedron, and their mean planes make a dihedral angle of $58.71(5)^{\circ}$. The amino groups bonded to $\mathrm{C} 8$ and $\mathrm{C} 18$ are trans to the cyanide ligands. The octahedral geometry around $\mathrm{Ag} 1$ is distorted, mainly because

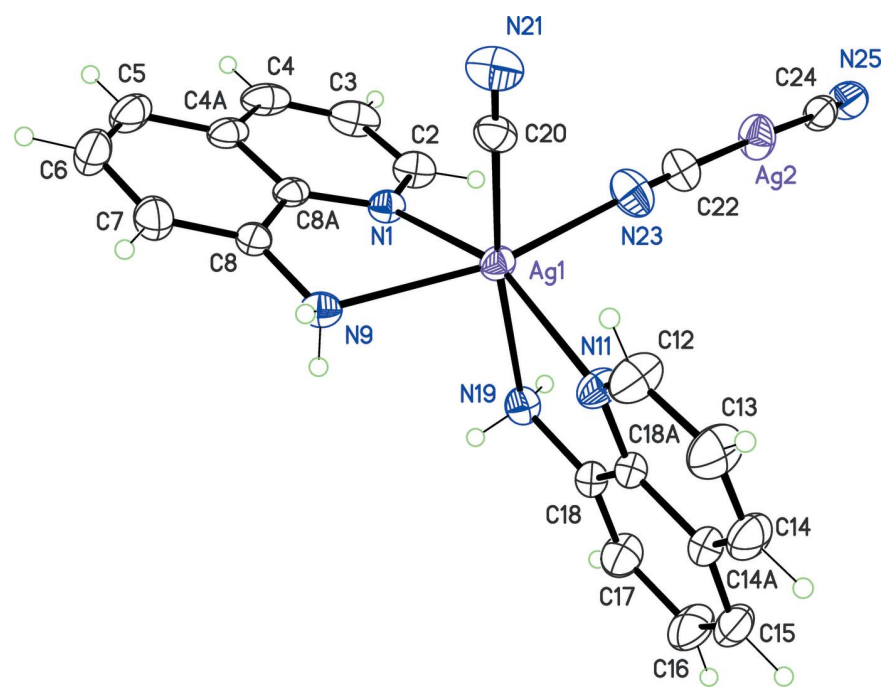

Figure 1

The molecular structure of the title complex, with displacement ellipsoids drawn at the $30 \%$ probability level.
Table 1

Hydrogen-bond geometry $\left(\AA{ }^{\circ}\right)$.

\begin{tabular}{lllll}
\hline$D-\mathrm{H} \cdots A$ & $D-\mathrm{H}$ & $\mathrm{H} \cdots A$ & $D \cdots A$ & $D-\mathrm{H} \cdots A$ \\
\hline $\mathrm{N} 9-\mathrm{H} 9 A \cdots \mathrm{N} 21^{\mathrm{i}}$ & $0.79(3)$ & $2.36(3)$ & $3.143(4)$ & $169(3)$ \\
$\mathrm{N} 9-\mathrm{H} 9 B \cdots \mathrm{N} 21^{\text {ii }}$ & $0.85(3)$ & $2.23(3)$ & $3.075(3)$ & $172(3)$ \\
$\mathrm{N} 19-\mathrm{H} 19 A \cdots \mathrm{N} 21^{\text {ii }}$ & $0.77(3)$ & $2.48(3)$ & $3.205(4)$ & $157(3)$ \\
$\mathrm{N} 19-\mathrm{H} 19 B \cdots \mathrm{N} 25^{\text {iii }}$ & $0.90(3)$ & $2.19(3)$ & $3.087(4)$ & $175(3)$ \\
\hline
\end{tabular}

Symmetry codes: (i) $\quad-x+1,-y+2,-z+1 ; \quad$ (ii) $\quad x, y-1, z$;

$-x+2, y-\frac{1}{2},-z+\frac{1}{2}$

of bite angles for quinoline ligands, $\mathrm{N} 1-\mathrm{Ag} 1-\mathrm{N} 9=69.59(7)^{\circ}$ and $\mathrm{N} 11-\mathrm{Ag} 1-\mathrm{N} 19=71.29(7)^{\circ}$. The coordination of the terminal cyanide ligand, $\mathrm{C} 20 \equiv \mathrm{N} 21$ is through the $\mathrm{C}$ atom, as determined from the structure refinement (see Refinement section). This orientation seems to be favored by the availability of atom N21 as an acceptor for hydrogen bonding with symmetry-related molecules in the crystal (Table 1).

Metal site Ag2 has a linear coordination with two cyanide ligands. Both ligands are coordinated through their $\mathrm{C}$ atoms (C22 and $\mathrm{C} 24)$, and the coordination angle $\mathrm{C} 22-\mathrm{Ag} 2-\mathrm{C} 24=$ $176.05(11)^{\circ}$, close to the ideal angle of $180^{\circ}$ expected for an $s p$ hybridization of the metal. Site Ag2 may thus be confidently assigned to a $\mathrm{Ag}^{\mathrm{I}}$ coordination site, and charge balance for the complex should then set the oxidation state for the octahedral metal as $\mathrm{Ag}^{\mathrm{II}}$, with a formal hybridization $s p^{3} d^{2}$. The title complex is a mixed-valence compound, with valences localized on a single site. According to the Robin-Day classification (Day et al., 2008), this compound should thus be a class 1 or class 2 mixed-valence compound. The deep-red color of the crystals should be the result of the $\pi^{*} \leftarrow 4 d(\mathrm{Ag})$ metal-toligand charge transfer, rather than a consequence of an intervalence charge transfer of a class 2 complex. Indeed, porphyrinato- $\mathrm{Ag}^{\mathrm{II}}$ compounds are generally purple or red compounds (e.g. Xu et al., 2007).

Cyanide ligand $\mathrm{C} 22 \equiv \mathrm{N} 23$ bridges metal sites $\mathrm{Ag} 1$ and $\mathrm{Ag} 2$, with oxidation states II and I respectively. The best structure refinement shows that this ligand is not disordered: the $\mathrm{C}$ atom is bonded to $\mathrm{Ag}^{+}$, and the $\mathrm{N}$ atom to the $\mathrm{Ag}^{\mathrm{II}}$ atom. This orientation observed for the bridge is consistent with the Pearson's HSAB principle (Pearson, 2005). The cyanide Lewis base is considered as a soft ligand, which preferentially forms covalent bonds with soft Lewis acid, like $\mathrm{Ag}^{+}$. However, the heteronuclear nature of this ligand induces an asymmetric character for the softness: based on the absolute electronegativity criterion, the $\mathrm{C}$ side of the cyanide ligand is expected to be softer than the $\mathrm{N}$ side. On the other hand, regarding the acid component of the coordination bonds, $\mathrm{Ag}^{+}$ is expected to be softer than $\mathrm{Ag}^{2+}$, due to the charge difference, which makes $\mathrm{Ag}^{+}$more polarizable than $\mathrm{Ag}^{2+}$. The most stable acid-base interactions for the bridging mode of ligand $\mathrm{C} 22 \equiv \mathrm{N} 23$ is thus $\mathrm{Ag}^{+}-\mathrm{C} \equiv \mathrm{N}-\mathrm{Ag}^{2+}$, as observed in the $\mathrm{X}$-ray-based structure refinement. From the reactivity point of view, the dicyanidoargentate(I) anion, $\left[\mathrm{Ag}(\mathrm{CN})_{2}\right]^{-}$, used as starting material, preserves the $\kappa \mathrm{C}$ coordination mode for the cyanide groups in the product. This anion thus acts as a ligand to the oxidized $\mathrm{Ag}^{\mathrm{II}}$ atom formed during the reaction. The 


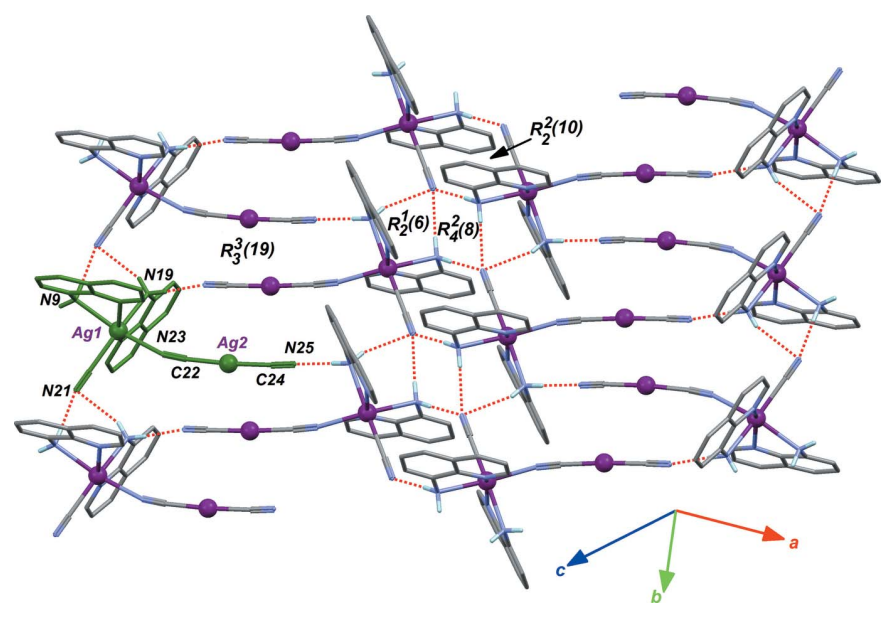

Figure 2

Part of the crystal structure of the title complex, emphasizing the $\mathrm{N}-$ $\mathrm{H} \cdots \mathrm{N}$ hydrogen bonds (dashed red lines) forming $R$ rings. The green molecule corresponds to the asymmetric unit.

same $\kappa \mathrm{C}$ coordination is observed for the terminal cyanide group bonded to $\mathrm{Ag}^{2+}$, indicating that this fragment $[\mathrm{Ag}(\mathrm{CN})]^{+}$is also produced from dicyanidoargentate, probably prior to aminoquinoline coordination.

\section{Supramolecular features}

As described in the previous section, both terminal cyanide ligands are bonded to $\mathrm{Ag} 1$ and $\mathrm{Ag} 2$ as $\kappa \mathrm{C}$ ligands, allowing the $\mathrm{N}$ terminus to act as acceptor sites for hydrogen bonding (Ramabhadran et al., 2014). Amino groups of aminoquinoline ligands are the donors for these contacts (Table 1), forming a two-dimensional supramolecular network parallel to (102) (Fig. 2). Molecules are aggregated through a centrosymmetric $R_{4}^{2}(8)$ ring, where the donor group is the terminal cyanide C20/ N21 bonded to Ag1. The same cyanide ligand is engaged in $R_{2}^{1}(6)$ rings, where donors are from two different amino groups. This basic pattern of fused rings propagates in the [010] direction, via larger $R_{2}^{2}(10)$ rings. Finally, these rows of molecules are connected in the crystal via the long arms Ag2$\mathrm{C} 24 \equiv \mathrm{N} 25$, which take part in large $R_{3}^{3}(19)$ rings. The shortest metal - metal distance is observed in these rings involving $\mathrm{Ag}^{+}$ions: $\mathrm{Ag} 2 \cdots \mathrm{Ag} 2^{\mathrm{i}}=3.9680$ (3) $\AA$ [symmetry code (i): $\left.-x+2, y+\frac{1}{2},-z+\frac{1}{2}\right]$.

Although the resulting supramolecular structure is compact, hydrogen bonds, with $\mathrm{H} \cdot \mathrm{N}$ contacts in the range 2.19 (3)-2.48 (3) $\AA$, should be considered as interactions of moderate strength. The crystallized compound is an authentic molecular complex, in which the terminal cyanide ligands are not engaged in polymeric bonds.

\section{Database survey}

Complexes characterized by X-ray diffraction which include at least one $\mathrm{Ag}^{2+}$ ion are much less common than $\mathrm{Ag}^{+}$complexes. An estimation using the field 'NAME = silver(II)' or 'NAME
$=$ silver(I)' in the current release of the CSD (version 5.36 with all updates; Groom \& Allen, 2014), affords 63 and more than 8000 hits, respectively. Within $\mathrm{Ag}^{\mathrm{I}}$ complexes, the occurrence of the dicyanidoargentate ion is significant. It has been used not only as a counter-ion (e.g. Stork et al., 2005) but also as a ligand for numerous transition-metal ions, including $\mathrm{Ag}^{+}$( $\mathrm{Lin}$ et al., 2005).

For non-polymeric compounds, the most common coordination for $\mathrm{Ag}^{2+}$ is the square-planar $\left[\mathrm{AgN}_{4}\right]$ arrangement, found in porphyrin derivatives and tetra-aza cyclic ligands (e.g. Xu et al., 2007). However, a few cases of sixcoordinate $\mathrm{Ag}^{2+}$ species have been characterized, with $N$-donor ligands (Clark et al., 2009) and $S$-donor ligands (Shaw et al., 2006). Compounds with both $\mathrm{Ag}^{+}$and $\mathrm{Ag}^{2+}$ ions which have been X-ray characterized seem to be very scarce. A 1D polymeric mixed-valent $\mathrm{Ag}^{\mathrm{I}} / \mathrm{Ag}^{\mathrm{II}}$ polymer was obtained by reacting $\mathrm{AgNO}_{3}, \mathrm{Na}_{2} \mathrm{~S}_{2} \mathrm{O}_{8}$ and pyrazine in a $\mathrm{CH}_{3} \mathrm{CN} / \mathrm{H}_{2} \mathrm{O}$ mixture, and the presence of $\mathrm{Ag}^{2+}$ was confirmed by ESR (Sun et al., 2010). The two other cases retrieved from the CSD are ionic compounds, in which tetraazacyclotetradecane derivatives coordinate the $\mathrm{Ag}^{2+}$ ion in a square-planar geometry, while the $\mathrm{Ag}^{+}$ion is present in the anionic polymeric part (Wang \& Mak, 2001) or in an anionic cluster (Wang et al., 2002). The title complex is, as far we can see, the first nonpolymeric and non-ionic mixed-valence $\mathrm{Ag}^{\mathrm{I}, \mathrm{II}}$ compound characterized by X-ray diffraction.

\section{Synthesis and crystallization}

The title compound was obtained under solvothermal conditions from a mixture of iron(II) sulfate heptahydrate $(28 \mathrm{mg}$, $0.1 \mathrm{mmol}$ ), quinolin- 8 -amine $(30 \mathrm{mg}, 0.2 \mathrm{mmol})$ and potassium dicyanidoargentate $(40 \mathrm{mg}, 0.2 \mathrm{mmol})$ in water-ethanol $(4: 1 v / v, 20 \mathrm{ml})$. The mixture was transferred to a Teflon-lined autoclave and heated at $423 \mathrm{~K}$ for $48 \mathrm{~h}$. The autoclave was then allowed to cool to ambient temperature. Deep-red crystals of the title compound were collected by filtration, washed with water and dried in air (yield 30\%).

\section{Refinement}

Crystal data, data collection and structure refinement details are summarized in Table 2. Special attention was paid to the accurate orientation for the three cyanide ligands in the asymmetric unit. For each $\mathrm{C} \equiv \mathrm{N}$ group, two refinements were carried out with each possible orientation, and the best model was retained on the basis of $R_{1}$ and $w R_{2}$ factors, and ADP for the $\mathrm{C}$ and $\mathrm{N}$ sites. For example, $w R_{2}$ for all data rises from $8.78 \%$ to $c a .9 .30 \%$ if one cyanide ligand bonded to $\mathrm{Ag} 2$ is inverted. No evidence for disordered cyanido groups was detected in the difference maps. All C-bonded $\mathrm{H}$ atoms were placed in calculated positions and refined as riding atoms, with $\mathrm{C}-\mathrm{H}$ bond lengths fixed to $0.93 \AA$. Amino $\mathrm{H}$ atoms bonded to N9 and N19 were found in a difference map and refined freely. For all $\mathrm{H}$ atoms, isotropic displacement parameters were calculated as $U_{\text {iso }}(\mathrm{H})=1.2 U_{\text {eq }}($ carrier atom $)$. 
Table 2

Experimental details.

\begin{tabular}{ll}
\hline Crystal data & \\
Chemical formula & {$\left[\mathrm{Ag}_{2}(\mathrm{CN})_{3}\left(\mathrm{C}_{9} \mathrm{H}_{8} \mathrm{~N}_{2}\right)_{2}\right]$} \\
$M_{\mathrm{r}}$ & 582.15 \\
Crystal system, space group & Monoclinic, $P 2_{1} / c$ \\
Temperature $(\mathrm{K})$ & 293 \\
$a, b, c(\AA)$ & $13.5449(7), 6.9385(3)$, \\
& $22.3824(11)$ \\
$\beta\left({ }^{\circ}\right)$ & $94.767(2)$ \\
$V\left(\AA^{3}\right)$ & $2096.25(17)$ \\
$Z$ & 4 \\
Radiation type & Mo $K \alpha$ \\
$\mu\left(\mathrm{mm}^{-1}\right)$ & 1.89 \\
Crystal size $(\mathrm{mm})$ & $0.27 \times 0.23 \times 0.18$ \\
& \\
Data collection & Bruker APEXII CCD \\
Diffractometer & Multi-scan $(S A D A B S ;$ Sheldrick, \\
Absorption correction & $2003)$ \\
& $0.615,0.754$ \\
$T_{\text {min }}, T_{\max }$ & $27103,7113,5226$ \\
No. of measured, independent and & \\
$\quad$ observed $[I>2 \sigma(I)]$ reflections & 0.021 \\
$R_{\text {int }}$ & 0.750 \\
$(\text { sin } \theta / \lambda)_{\text {max }}\left(\AA^{-1}\right)$ & \\
Refinement & \\
$R\left[F^{2}>2 \sigma\left(F^{2}\right)\right], w R\left(F^{2}\right), S$ & $0.034,0.088,1.02$ \\
No. of reflections & 7113 \\
No. of parameters & 283 \\
H-atom treatment & H atoms treated by a mixture of \\
& independent and constrained \\
$\Delta \rho_{\text {max }}, \Delta \rho_{\text {min }}\left(\mathrm{e} \AA^{-3}\right)$ & refinement \\
\hline
\end{tabular}

Computer programs: APEX2 and SAINT (Bruker, 2009), SHELXS2014/7 (Sheldrick, 2008), SHELXL2014/7 (Sheldrick, 2015) and Mercury (Macrae et al., 2008).

\section{Acknowledgements}

The authors acknowledge the Algerian Ministry of Higher Education and Scientific Research, the Algerian Directorate General for Scientific Research and Technological Development and Ferhat Abbas Sétif 1 University for financial support.

\section{References}

Atmani, C., Setifi, F., Benmansour, S., Triki, S., Marchivie, M., Salaün, J.-Y. \& Gómez-García, C. J. (2008). Inorg. Chem. Commun. 11, 921-924.

Benmansour, S., Setifi, F., Gómez-García, C. J., Triki, S. \& Coronado, E. (2008). Inorg. Chim. Acta, 361, 3856-3862.

Benmansour, S., Setifi, F., Triki, S. \& Gómez-García, C. J. (2012). Inorg. Chem. 51, 2359-2365.

Benmansour, S., Setifi, F., Triki, S., Thétiot, F., Sala-Pala, J., GómezGarcía, C. J. \& Colacio, E. (2009). Polyhedron, 28, 1308-1314.

Bruker (2009). APEX2 and SAINT. Bruker AXS Inc., Madison, Wisconsin, USA.
Clark, I. J., Crispini, A., Donnelly, P. S., Engelhardt, L. M., Harrowfield, J. M., Jeong, S.-H., Kim, Y., Koutsantonis, G. A., Lee, Y. H., Lengkeek, N. A., Mocerino, M., Nealon, G. L., Ogden, M. I., Park, Y. C., Pettinari, C., Polanzan, L., Rukmini, E., Sargeson, A. M., Skelton, B. W., Sobolev, A. N., Thuéry, P. \& White, A. H. (2009). Aust. J. Chem. 62, 1246-1260.

Day, P., Hush, N. S. \& Clark, R. J. H. (2008). Philos. Trans. R. Soc. London A, 366, 5-14.

Dupouy, G., Marchivie, M., Triki, S., Sala-Pala, J., Gómez-García, C. J., Pillet, S., Lecomte, C. \& Létard, J.-F. (2009). Chem. Commun. pp. 3404-3406.

Dupouy, G., Marchivie, M., Triki, S., Sala-Pala, J., Salaün, J.-Y., Gómez-García, C. J. \& Guionneau, P. (2008). Inorg. Chem. 47, 8921-8931.

Grochala, W. \& Mazej, Z. (2015). Philos. Trans. Roy. Soc. A: Math. Phys. Engineering Sci. 373, 20140179.

Groom, C. R. \& Allen, F. H. (2014). Angew. Chem. Int. Ed. 53, 662671.

Housecroft, C. E. \& Sharpe, A. G. (2012). Inorg. Chem. 4th ed., ch. 22. Harlow: Pearson.

Kandaiah, S., Huebner, R. \& Jansen, M. (2012). Polyhedron, 48, 6871.

Lin, Y.-Y., Lai, S.-W., Che, C.-M., Fu, W.-F., Zhou, Z.-Y. \& Zhu, N. (2005). Inorg. Chem. 44, 1511-1524.

Macrae, C. F., Bruno, I. J., Chisholm, J. A., Edgington, P. R., McCabe, P., Pidcock, E., Rodriguez-Monge, L., Taylor, R., van de Streek, J. \& Wood, P. A. (2008). J. Appl. Cryst. 41, 466-470.

Pearson, R. G. (2005). J. Chem. Sci. 117, 369-377.

Ramabhadran, R. O., Hua, Y., Flood, A. H. \& Raghavachari, K. (2014). J. Phys. Chem. A, 118, 7418-7423.

Setifi, F., Benmansour, S., Marchivie, M., Dupouy, G., Triki, S., SalaPala, J., Salaün, J.-Y., Gómez-García, C. J., Pillet, S., Lecomte, C. \& Ruiz, E. (2009). Inorg. Chem. 48, 1269-1271.

Setifi, F., Charles, C., Houille, S., Thétiot, T., Triki, S., Gómez-García, C. J. \& Pillet, S. (2014). Polyhedron, 61, 242-247.

Setifi, Z., Domasevitch, K. V., Setifi, F., Mach, P., Ng, S. W., Petř́iček, V. \& Dušek, M. (2013). Acta Cryst. C69, 1351-1356.

Setifi, Z., Lehchili, F., Setifi, F., Beghidja, A., Ng, S. W. \& Glidewell, C. (2014). Acta Cryst. C70, 338-341.

Setifi, F., Milin, E., Charles, C., Thétiot, F., Triki, S. \& Gómez-García, C. J. (2014). Inorg. Chem. 53, 97-104.

Shaw, J. L., Wolowska, J., Collison, D., Howard, J. A. K., McInnes, E. J. L., McMaster, J., Blake, A. J., Wilson, C. \& Schröder, M. (2006). J. Am. Chem. Soc. 128, 13827-13839.

Sheldrick, G. M. (2003). SADABS. University of Göttingen, Germany.

Sheldrick, G. M. (2008). Acta Cryst. A64, 112-122.

Sheldrick, G. M. (2015). Acta Cryst. C71, 3-8.

Stork, J. R., Rios, D., Pham, D., Bicocca, V., Olmstead, M. M. \& Balch, A. L. (2005). Inorg. Chem. 44, 3466-3472.

Sun, D., Yang, C.-F., Xu, H.-R., Zhao, H.-X., Wei, Z.-H., Zhang, N., Yu, L.-J., Huang, R.-B. \& Zheng, L.-S. (2010). Chem. Commun. 46, 8168-8170.

Wang, Q.-M., Lee, H. K. \& Mak, T. C. W. (2002). New J. Chem. 26, 513-515.

Wang, Q.-M. \& Mak, T. C. W. (2001). Chem. Commun. pp. 807-808.

Xu, Y.-J., Yang, X.-X., Cao, H. \& Zhao, H.-B. (2007). Acta Cryst. E63, m1437. 


\section{supporting information}

Acta Cryst. (2015). E71, 698-701 [doi:10.1107/S2056989015009664]

Crystal structure of $\mu$-cyanido-1:2 $\kappa^{2} \mathrm{~N}: C$-dicyanido- $1 \kappa C, 2 \kappa C$-bis(quinolin-8amine-1 $\left.\kappa^{2} N, N^{\prime}\right)$-2-silver(I)-1-silver(II): rare occurrence of a mixed-valence $\mathbf{A g}^{\prime \prime \prime I I}$ compound

\section{Zouaoui Setifi, Sylvain Bernès, Olivier Pérez, Fatima Setifi and Djamil-Azzeddine Rouag}

Computing details

Data collection: APEX2 (Bruker, 2009); cell refinement: APEX2 and SAINT (Bruker, 2009); data reduction: SAINT

(Bruker, 2009); program(s) used to solve structure: SHELXS2014/7 (Sheldrick, 2008); program(s) used to refine structure: SHELXL2014/7 (Sheldrick, 2015); molecular graphics: Mercury (Macrae et al., 2008); software used to prepare material for publication: SHELXL2014/7 (Sheldrick, 2015).

$\mu$-Cyanido-1:2 $\kappa^{2} N$ :C-dicyanido-1 $\kappa C, 2 \kappa C$-bis(quinolin-8-amine-1 $\left.\kappa^{2} N, N^{\prime}\right)$-2-silver(I)-1-silver(II)

Crystal data

$\left[\mathrm{Ag}_{2}(\mathrm{CN})_{3}\left(\mathrm{C}_{9} \mathrm{H}_{8} \mathrm{~N}_{2}\right)_{2}\right]$

$M_{r}=582.15$

Monoclinic, $P 2_{1} / c$

$a=13.5449(7) \AA$

$b=6.9385(3) \AA$

$c=22.3824$ (11) $\AA$

$\beta=94.767(2)^{\circ}$

$V=2096.25(17) \AA^{3}$

$Z=4$

$F(000)=1140$

$D_{\mathrm{x}}=1.845 \mathrm{Mg} \mathrm{m}^{-3}$

Mo $K \alpha$ radiation, $\lambda=0.71073 \AA$

Cell parameters from 9889 reflections

$\theta=3.1-30.7^{\circ}$

$\mu=1.89 \mathrm{~mm}^{-1}$

$T=293 \mathrm{~K}$

Prism, deep-red

$0.27 \times 0.23 \times 0.18 \mathrm{~mm}$

Data collection

Bruker APEXII CCD

7113 independent reflections

diffractometer

Radiation source: fine-focus sealed tube

$\varphi \& \omega$ scans

5226 reflections with $I>2 \sigma(I)$

$R_{\text {int }}=0.021$

$\theta_{\text {max }}=32.2^{\circ}, \theta_{\min }=4.2^{\circ}$

Absorption correction: multi-scan

$h=-20 \rightarrow 17$

(SADABS; Sheldrick, 2003)

$T_{\min }=0.615, T_{\max }=0.754$

$k=-7 \rightarrow 10$

$l=-33 \rightarrow 32$

27103 measured reflections

\section{Refinement}

Refinement on $F^{2}$

Least-squares matrix: full

$R\left[F^{2}>2 \sigma\left(F^{2}\right)\right]=0.034$

$w R\left(F^{2}\right)=0.088$

$S=1.02$

7113 reflections

283 parameters

0 restraints

0 constraints

Hydrogen site location: mixed

$\mathrm{H}$ atoms treated by a mixture of independent and constrained refinement

$w=1 /\left[\sigma^{2}\left(F_{\mathrm{o}}^{2}\right)+(0.043 P)^{2}+0.5603 P\right]$

where $P=\left(F_{\mathrm{o}}{ }^{2}+2 F_{\mathrm{c}}{ }^{2}\right) / 3$ 
$(\Delta / \sigma)_{\max }=0.001$

$\Delta \rho_{\max }=1.70$ e $\AA^{-3}$

$\Delta \rho_{\min }=-0.56$ e $\AA^{-3}$

Fractional atomic coordinates and isotropic or equivalent isotropic displacement parameters $\left(\AA^{2}\right)$

\begin{tabular}{|c|c|c|c|c|}
\hline & $x$ & $y$ & $z$ & $U_{\text {iso }} * / U_{\text {eq }}$ \\
\hline Ag1 & $0.66686(2)$ & $0.78323(2)$ & $0.41853(2)$ & $0.03575(6)$ \\
\hline Ag2 & $0.97403(2)$ & $0.95903(4)$ & $0.28877(2)$ & $0.05800(8)$ \\
\hline N1 & $0.58984(15)$ & $0.6944(3)$ & $0.32264(9)$ & $0.0372(4)$ \\
\hline $\mathrm{C} 2$ & $0.6314(2)$ & $0.7024(4)$ & $0.27131(12)$ & $0.0487(6)$ \\
\hline $\mathrm{H} 2 \mathrm{~A}$ & 0.7001 & 0.7094 & 0.2722 & $0.058 *$ \\
\hline $\mathrm{C} 3$ & $0.5762(3)$ & $0.7006(4)$ & $0.21569(13)$ & $0.0593(8)$ \\
\hline $\mathrm{H} 3 \mathrm{~A}$ & 0.6080 & 0.7002 & 0.1804 & $0.071^{*}$ \\
\hline $\mathrm{C} 4$ & $0.4768(3)$ & $0.6994(4)$ & $0.21363(13)$ & $0.0584(8)$ \\
\hline $\mathrm{H} 4 \mathrm{~A}$ & 0.4397 & 0.7024 & 0.1768 & $0.070 *$ \\
\hline $\mathrm{C} 4 \mathrm{~A}$ & $0.4286(2)$ & $0.6937(3)$ & $0.26690(12)$ & $0.0470(6)$ \\
\hline $\mathrm{C} 5$ & $0.3257(2)$ & $0.6965(4)$ & $0.26830(17)$ & $0.0631(9)$ \\
\hline $\mathrm{H} 5 \mathrm{~A}$ & 0.2852 & 0.7052 & 0.2327 & $0.076^{*}$ \\
\hline C6 & $0.2850(2)$ & $0.6865(4)$ & $0.32116(19)$ & $0.0682(9)$ \\
\hline H6A & 0.2166 & 0.6928 & 0.3218 & $0.082 *$ \\
\hline $\mathrm{C} 7$ & $0.3441(2)$ & $0.6670(4)$ & $0.37504(15)$ & $0.0552(7)$ \\
\hline H7A & 0.3142 & 0.6564 & 0.4109 & $0.066^{*}$ \\
\hline $\mathrm{C} 8$ & $0.44478(18)$ & $0.6633(3)$ & $0.37580(11)$ & $0.0382(5)$ \\
\hline C8A & $0.48939(18)$ & $0.6834(3)$ & $0.32138(11)$ & $0.0360(5)$ \\
\hline N9 & $0.50821(17)$ & $0.6373(3)$ & $0.42895(10)$ & $0.0400(5)$ \\
\hline H9A & $0.481(2)$ & $0.675(4)$ & $0.4567(14)$ & $0.048^{*}$ \\
\hline H9B & $0.525(2)$ & $0.519(4)$ & $0.4314(13)$ & $0.048^{*}$ \\
\hline N11 & $0.74947(17)$ & $0.7366(3)$ & $0.51431(9)$ & $0.0445(5)$ \\
\hline $\mathrm{C} 12$ & $0.7454(2)$ & $0.8572(4)$ & $0.55930(13)$ & $0.0617(8)$ \\
\hline $\mathrm{H} 12 \mathrm{~A}$ & 0.7000 & 0.9578 & 0.5554 & $0.074 *$ \\
\hline C13 & $0.8057(3)$ & $0.8419(5)$ & $0.61242(14)$ & $0.0682(9)$ \\
\hline $\mathrm{H} 13 \mathrm{~A}$ & 0.8014 & 0.9322 & 0.6428 & $0.082 *$ \\
\hline C14 & $0.8705(2)$ & $0.6948(4)$ & $0.61937(14)$ & $0.0597(8)$ \\
\hline $\mathrm{H} 14 \mathrm{~A}$ & 0.9111 & 0.6831 & 0.6548 & $0.072 *$ \\
\hline C14A & $0.87684(19)$ & $0.5588(4)$ & $0.57329(12)$ & $0.0448(6)$ \\
\hline C15 & $0.9406(2)$ & $0.4006(4)$ & $0.57738(15)$ & $0.0590(8)$ \\
\hline $\mathrm{H} 15 \mathrm{~A}$ & 0.9829 & 0.3824 & 0.6118 & $0.071^{*}$ \\
\hline C16 & $0.9414(3)$ & $0.2742(4)$ & $0.53187(18)$ & $0.0708(10)$ \\
\hline H16A & 0.9836 & 0.1684 & 0.5354 & $0.085^{*}$ \\
\hline C17 & $0.8793(2)$ & $0.3000(4)$ & $0.47926(14)$ & $0.0560(7)$ \\
\hline H17A & 0.8815 & 0.2114 & 0.4482 & $0.067 *$ \\
\hline C18 & $0.81619(17)$ & 0.4517 & $0.47276(11)$ & $0.0379(5)$ \\
\hline C18A & $0.81318(16)$ & 0.5856 & $0.52035(10)$ & $0.0362(5)$ \\
\hline N19 & $0.75194(17)$ & 0.4838 & $0.41939(10)$ & $0.0404(5)$ \\
\hline H19A & $0.716(2)$ & $0.397(4)$ & $0.4210(12)$ & $0.048^{*}$ \\
\hline H19B & $0.788(2)$ & $0.478(4)$ & $0.3873(14)$ & $0.048^{*}$ \\
\hline $\mathrm{C} 20$ & $0.6010(2)$ & $1.0782(4)$ & $0.44098(11)$ & $0.0431(6)$ \\
\hline $\mathrm{N} 21$ & $0.5709(2)$ & $1.2156(4)$ & $0.45109(12)$ & $0.0620(7)$ \\
\hline
\end{tabular}




\begin{tabular}{lllll}
$\mathrm{C} 22$ & $0.8639(2)$ & $0.9377(4)$ & $0.34572(14)$ & $0.0525(7)$ \\
$\mathrm{N} 23$ & $0.8017(2)$ & $0.9129(4)$ & $0.37489(12)$ & $0.0607(6)$ \\
$\mathrm{C} 24$ & $1.0814(2)$ & $0.9611(4)$ & $0.23044(13)$ & $0.0497(6)$ \\
$\mathrm{N} 25$ & $1.1367(2)$ & $0.9559(4)$ & $0.19515(12)$ & $0.0620(7)$ \\
\hline
\end{tabular}

Atomic displacement parameters $\left(\AA^{2}\right)$

\begin{tabular}{|c|c|c|c|c|c|c|}
\hline & $U^{11}$ & $U^{22}$ & $U^{33}$ & $U^{12}$ & $U^{13}$ & $U^{23}$ \\
\hline Ag1 & $0.03820(10)$ & $0.03710(10)$ & $0.03135(10)$ & $0.00115(7)$ & $-0.00073(7)$ & $-0.00062(7)$ \\
\hline Ag2 & $0.04638(14)$ & $0.07771(16)$ & $0.05085(14)$ & $-0.00800(10)$ & $0.00974(10)$ & $0.00022(10)$ \\
\hline N1 & $0.0449(11)$ & $0.0354(10)$ & $0.0311(10)$ & $0.0019(8)$ & $0.0019(8)$ & $-0.0006(7)$ \\
\hline $\mathrm{C} 2$ & $0.0625(17)$ & $0.0462(14)$ & $0.0379(14)$ & $0.0028(12)$ & $0.0082(12)$ & $-0.0016(11)$ \\
\hline $\mathrm{C} 3$ & $0.101(3)$ & $0.0448(15)$ & $0.0322(14)$ & $0.0020(15)$ & $0.0083(15)$ & $-0.0017(11)$ \\
\hline $\mathrm{C} 4$ & $0.096(3)$ & $0.0372(14)$ & $0.0383(15)$ & $0.0027(14)$ & $-0.0184(15)$ & $-0.0002(11)$ \\
\hline $\mathrm{C} 4 \mathrm{~A}$ & $0.0630(17)$ & $0.0279(11)$ & $0.0464(15)$ & $0.0007(10)$ & $-0.0183(13)$ & $-0.0006(10)$ \\
\hline $\mathrm{C} 5$ & $0.0601(19)$ & $0.0490(16)$ & $0.074(2)$ & $0.0009(13)$ & $-0.0320(17)$ & $0.0012(14)$ \\
\hline C6 & $0.0419(17)$ & $0.0603(19)$ & $0.099(3)$ & $-0.0001(13)$ & $-0.0157(18)$ & $0.0021(17)$ \\
\hline $\mathrm{C} 7$ & $0.0449(15)$ & $0.0506(14)$ & $0.071(2)$ & $-0.0012(12)$ & $0.0079(14)$ & $0.0014(14)$ \\
\hline $\mathrm{C} 8$ & $0.0390(13)$ & $0.0296(10)$ & $0.0455(14)$ & $0.0001(9)$ & $-0.0001(10)$ & $0.0012(9)$ \\
\hline $\mathrm{C} 8 \mathrm{~A}$ & $0.0457(13)$ & $0.0225(9)$ & $0.0383(13)$ & $0.0021(8)$ & $-0.0057(10)$ & $-0.0014(8)$ \\
\hline N9 & $0.0472(12)$ & $0.0381(11)$ & $0.0355(11)$ & $0.0048(9)$ & $0.0083(9)$ & $0.0019(9)$ \\
\hline N11 & $0.0491(13)$ & $0.0469(11)$ & $0.0359(11)$ & $0.0087(9)$ & $-0.0062(9)$ & $-0.0054(9)$ \\
\hline $\mathrm{C} 12$ & $0.074(2)$ & $0.0592(17)$ & $0.0497(17)$ & $0.0210(15)$ & $-0.0114(15)$ & $-0.0173(14)$ \\
\hline $\mathrm{C} 13$ & $0.089(2)$ & $0.0686(19)$ & $0.0438(17)$ & $0.0094(18)$ & $-0.0156(16)$ & $-0.0194(15)$ \\
\hline $\mathrm{C} 14$ & $0.068(2)$ & $0.0594(17)$ & $0.0474(17)$ & $0.0005(14)$ & $-0.0217(14)$ & $-0.0051(13)$ \\
\hline $\mathrm{C} 14 \mathrm{~A}$ & $0.0402(13)$ & $0.0473(14)$ & $0.0450(15)$ & $-0.0041(10)$ & $-0.0074(11)$ & $0.0028(11)$ \\
\hline $\mathrm{C} 15$ & $0.0513(17)$ & $0.0565(16)$ & $0.065(2)$ & $0.0051(13)$ & $-0.0196(14)$ & $0.0039(15)$ \\
\hline $\mathrm{C} 16$ & $0.064(2)$ & $0.0536(17)$ & 0.091 & $0.0210(14)$ & $-0.0199(18)$ & $-0.0047(17)$ \\
\hline $\mathrm{C} 17$ & $0.0556(17)$ & $0.0464(15)$ & $0.0643(19)$ & $0.0098(12)$ & $-0.0041(14)$ & $-0.0121(13)$ \\
\hline $\mathrm{C} 18$ & $0.0334(12)$ & $0.0390(12)$ & $0.0408(13)$ & $-0.0025(9)$ & $0.0003(10)$ & $0.0000(9)$ \\
\hline C18A & $0.0316(11)$ & $0.0386(11)$ & $0.0376(13)$ & $-0.0018(9)$ & $-0.0010(9)$ & $0.0017(9)$ \\
\hline N19 & $0.0422(12)$ & $0.0432(11)$ & $0.0359(11)$ & $-0.0028(8)$ & $0.0041(9)$ & $-0.0047(9)$ \\
\hline $\mathrm{C} 20$ & $0.0539(15)$ & $0.0388(13)$ & $0.0365(13)$ & $-0.0039(11)$ & $0.0025(11)$ & $0.0045(10)$ \\
\hline $\mathrm{N} 21$ & $0.0785(19)$ & $0.0514(14)$ & $0.0581(16)$ & $0.0082(13)$ & $0.0184(14)$ & $0.0060(12)$ \\
\hline $\mathrm{C} 22$ & $0.0526(17)$ & $0.0500(15)$ & $0.0553(18)$ & $-0.0087(12)$ & $0.0074(14)$ & $-0.0033(12)$ \\
\hline $\mathrm{N} 23$ & $0.0626(16)$ & $0.0563(14)$ & $0.0659(17)$ & $-0.0120(12)$ & $0.0221(13)$ & $-0.0064(12)$ \\
\hline $\mathrm{C} 24$ & $0.0423(15)$ & $0.0592(16)$ & $0.0470(16)$ & $0.0007(12)$ & $-0.0004(13)$ & $0.0082(12)$ \\
\hline N25 & $0.0548(15)$ & $0.0745(17)$ & $0.0581(17)$ & $0.0054(12)$ & $0.0119(13)$ & $0.0138(12)$ \\
\hline
\end{tabular}

Geometric parameters $\left(\AA,{ }^{\circ}\right)$

\begin{tabular}{llll}
\hline $\mathrm{Ag} 1-\mathrm{C} 20$ & $2.305(3)$ & $\mathrm{N} 9-\mathrm{H} 9 \mathrm{~A}$ & $0.79(3)$ \\
$\mathrm{Ag} 1-\mathrm{N} 23$ & $2.323(3)$ & $\mathrm{N} 9-\mathrm{H} 9 \mathrm{~B}$ & $0.85(3)$ \\
$\mathrm{Ag} 1-\mathrm{N} 11$ & $2.357(2)$ & $\mathrm{N} 11-\mathrm{C} 12$ & $1.314(3)$ \\
$\mathrm{Ag} 1-\mathrm{N} 19$ & $2.375(2)$ & $\mathrm{N} 11-\mathrm{C} 18 \mathrm{~A}$ & $1.357(3)$ \\
$\mathrm{Ag} 1-\mathrm{N} 1$ & $2.3878(19)$ & $\mathrm{C} 12-\mathrm{C} 13$ & $1.389(4)$ \\
$\mathrm{Ag} 1-\mathrm{N} 9$ & $2.404(2)$ & $\mathrm{C} 12-\mathrm{H} 12 \mathrm{~A}$ & 0.9300 \\
$\mathrm{Ag} 2-\mathrm{C} 24$ & $2.033(3)$ & $\mathrm{C} 13-\mathrm{C} 14$ & $1.347(4)$
\end{tabular}




\begin{tabular}{|c|c|c|c|}
\hline $\mathrm{Ag} 2-\mathrm{C} 22$ & $2.047(3)$ & $\mathrm{C} 13-\mathrm{H} 13 \mathrm{~A}$ & 0.9300 \\
\hline $\mathrm{N} 1-\mathrm{C} 2$ & $1.322(3)$ & $\mathrm{C} 14-\mathrm{C} 14 \mathrm{~A}$ & $1.406(4)$ \\
\hline $\mathrm{N} 1-\mathrm{C} 8 \mathrm{~A}$ & $1.361(3)$ & $\mathrm{C} 14-\mathrm{H} 14 \mathrm{~A}$ & 0.9300 \\
\hline $\mathrm{C} 2-\mathrm{C} 3$ & $1.398(4)$ & $\mathrm{C} 14 \mathrm{~A}-\mathrm{C} 15$ & $1.395(4)$ \\
\hline $\mathrm{C} 2-\mathrm{H} 2 \mathrm{~A}$ & 0.9300 & $\mathrm{C} 14 \mathrm{~A}-\mathrm{C} 18 \mathrm{~A}$ & $1.419(3)$ \\
\hline $\mathrm{C} 3-\mathrm{C} 4$ & $1.343(5)$ & $\mathrm{C} 15-\mathrm{C} 16$ & $1.345(5)$ \\
\hline $\mathrm{C} 3-\mathrm{H} 3 \mathrm{~A}$ & 0.9300 & C15-H15A & 0.9300 \\
\hline $\mathrm{C} 4-\mathrm{C} 4 \mathrm{~A}$ & $1.407(4)$ & $\mathrm{C} 16-\mathrm{C} 17$ & $1.400(4)$ \\
\hline $\mathrm{C} 4-\mathrm{H} 4 \mathrm{~A}$ & 0.9300 & $\mathrm{C} 16-\mathrm{H} 16 \mathrm{~A}$ & 0.9300 \\
\hline $\mathrm{C} 4 \mathrm{~A}-\mathrm{C} 5$ & $1.397(4)$ & $\mathrm{C} 17-\mathrm{C} 18$ & $1.357(3)$ \\
\hline $\mathrm{C} 4 \mathrm{~A}-\mathrm{C} 8 \mathrm{~A}$ & $1.415(3)$ & C17-H17A & 0.9300 \\
\hline $\mathrm{C} 5-\mathrm{C} 6$ & $1.347(5)$ & $\mathrm{C} 18-\mathrm{C} 18 \mathrm{~A}$ & $1.417(3)$ \\
\hline $\mathrm{C} 5-\mathrm{H} 5 \mathrm{~A}$ & 0.9300 & $\mathrm{C} 18-\mathrm{N} 19$ & $1.436(3)$ \\
\hline $\mathrm{C} 6-\mathrm{C} 7$ & $1.397(5)$ & N19-H19A & $0.77(3)$ \\
\hline $\mathrm{C} 6-\mathrm{H} 6 \mathrm{~A}$ & 0.9300 & N19-H19B & $0.90(3)$ \\
\hline $\mathrm{C} 7-\mathrm{C} 8$ & $1.363(4)$ & $\mathrm{C} 20-\mathrm{N} 21$ & $1.069(3)$ \\
\hline $\mathrm{C} 7-\mathrm{H} 7 \mathrm{~A}$ & 0.9300 & $\mathrm{C} 22-\mathrm{N} 23$ & $1.121(4)$ \\
\hline $\mathrm{C} 8-\mathrm{C} 8 \mathrm{~A}$ & $1.411(4)$ & $\mathrm{C} 24-\mathrm{N} 25$ & $1.133(4)$ \\
\hline C8-N9 & $1.420(3)$ & & \\
\hline $\mathrm{C} 20-\mathrm{Ag} 1-\mathrm{N} 23$ & $94.56(9)$ & C8-N9-Ag1 & $110.41(15)$ \\
\hline $\mathrm{C} 20-\mathrm{Ag} 1-\mathrm{N} 11$ & $94.96(8)$ & $\mathrm{C} 8-\mathrm{N} 9-\mathrm{H} 9 \mathrm{~A}$ & $109(2)$ \\
\hline $\mathrm{N} 23-\mathrm{Ag} 1-\mathrm{N} 11$ & $96.04(9)$ & $\mathrm{Ag} 1-\mathrm{N} 9-\mathrm{H} 9 \mathrm{~A}$ & $114(2)$ \\
\hline $\mathrm{C} 20-\mathrm{Ag} 1-\mathrm{N} 19$ & $166.25(8)$ & $\mathrm{C} 8-\mathrm{N} 9-\mathrm{H} 9 \mathrm{~B}$ & $108.5(19)$ \\
\hline N23-Ag1-N19 & $86.81(9)$ & $\mathrm{Ag} 1-\mathrm{N} 9-\mathrm{H} 9 \mathrm{~B}$ & $100.1(19)$ \\
\hline N11-Ag1-N19 & $71.29(7)$ & $\mathrm{H} 9 \mathrm{~A}-\mathrm{N} 9-\mathrm{H} 9 \mathrm{~B}$ & $114(3)$ \\
\hline $\mathrm{C} 20-\mathrm{Ag} 1-\mathrm{N} 1$ & $106.09(8)$ & $\mathrm{C} 12-\mathrm{N} 11-\mathrm{C} 18 \mathrm{~A}$ & $118.8(2)$ \\
\hline $\mathrm{N} 23-\mathrm{Ag} 1-\mathrm{N} 1$ & $91.27(8)$ & $\mathrm{C} 12-\mathrm{N} 11-\mathrm{Ag} 1$ & $124.28(18)$ \\
\hline $\mathrm{N} 11-\mathrm{Ag} 1-\mathrm{N} 1$ & $157.10(7)$ & $\mathrm{C} 18 \mathrm{~A}-\mathrm{N} 11-\mathrm{Ag} 1$ & $116.52(16)$ \\
\hline $\mathrm{N} 19-\mathrm{Ag} 1-\mathrm{N} 1$ & $87.54(7)$ & $\mathrm{N} 11-\mathrm{C} 12-\mathrm{C} 13$ & $123.3(3)$ \\
\hline $\mathrm{C} 20-\mathrm{Ag} 1-\mathrm{N} 9$ & $89.30(9)$ & $\mathrm{N} 11-\mathrm{C} 12-\mathrm{H} 12 \mathrm{~A}$ & 118.4 \\
\hline $\mathrm{N} 23-\mathrm{Ag} 1-\mathrm{N} 9$ & $160.78(9)$ & $\mathrm{C} 13-\mathrm{C} 12-\mathrm{H} 12 \mathrm{~A}$ & 118.4 \\
\hline N11-Ag1—N9 & $102.39(8)$ & $\mathrm{C} 14-\mathrm{C} 13-\mathrm{C} 12$ & $119.2(3)$ \\
\hline N19-Ag1—N9 & $93.89(8)$ & $\mathrm{C} 14-\mathrm{C} 13-\mathrm{H} 13 \mathrm{~A}$ & 120.4 \\
\hline N1—Ag1—N9 & $69.59(7)$ & $\mathrm{C} 12-\mathrm{C} 13-\mathrm{H} 13 \mathrm{~A}$ & 120.4 \\
\hline $\mathrm{C} 24-\mathrm{Ag} 2-\mathrm{C} 22$ & $176.05(11)$ & $\mathrm{C} 13-\mathrm{C} 14-\mathrm{C} 14 \mathrm{~A}$ & $120.2(3)$ \\
\hline $\mathrm{C} 2-\mathrm{N} 1-\mathrm{C} 8 \mathrm{~A}$ & $118.8(2)$ & $\mathrm{C} 13-\mathrm{C} 14-\mathrm{H} 14 \mathrm{~A}$ & 119.9 \\
\hline $\mathrm{C} 2-\mathrm{N} 1-\mathrm{Ag} 1$ & $125.78(18)$ & $\mathrm{C} 14 \mathrm{~A}-\mathrm{C} 14-\mathrm{H} 14 \mathrm{~A}$ & 119.9 \\
\hline $\mathrm{C} 8 \mathrm{~A}-\mathrm{N} 1-\mathrm{Ag} 1$ & $113.24(15)$ & $\mathrm{C} 15-\mathrm{C} 14 \mathrm{~A}-\mathrm{C} 14$ & $123.7(3)$ \\
\hline $\mathrm{N} 1-\mathrm{C} 2-\mathrm{C} 3$ & $122.6(3)$ & $\mathrm{C} 15-\mathrm{C} 14 \mathrm{~A}-\mathrm{C} 18 \mathrm{~A}$ & $119.2(2)$ \\
\hline $\mathrm{N} 1-\mathrm{C} 2-\mathrm{H} 2 \mathrm{~A}$ & 118.7 & $\mathrm{C} 14-\mathrm{C} 14 \mathrm{~A}-\mathrm{C} 18 \mathrm{~A}$ & $117.1(2)$ \\
\hline $\mathrm{C} 3-\mathrm{C} 2-\mathrm{H} 2 \mathrm{~A}$ & 118.7 & $\mathrm{C} 16-\mathrm{C} 15-\mathrm{C} 14 \mathrm{~A}$ & $120.5(3)$ \\
\hline $\mathrm{C} 4-\mathrm{C} 3-\mathrm{C} 2$ & $119.4(3)$ & $\mathrm{C} 16-\mathrm{C} 15-\mathrm{H} 15 \mathrm{~A}$ & 119.8 \\
\hline $\mathrm{C} 4-\mathrm{C} 3-\mathrm{H} 3 \mathrm{~A}$ & 120.3 & $\mathrm{C} 14 \mathrm{~A}-\mathrm{C} 15-\mathrm{H} 15 \mathrm{~A}$ & 119.8 \\
\hline $\mathrm{C} 2-\mathrm{C} 3-\mathrm{H} 3 \mathrm{~A}$ & 120.3 & $\mathrm{C} 15-\mathrm{C} 16-\mathrm{C} 17$ & $120.8(3)$ \\
\hline $\mathrm{C} 3-\mathrm{C} 4-\mathrm{C} 4 \mathrm{~A}$ & $120.4(3)$ & $\mathrm{C} 15-\mathrm{C} 16-\mathrm{H} 16 \mathrm{~A}$ & 119.6 \\
\hline $\mathrm{C} 3-\mathrm{C} 4-\mathrm{H} 4 \mathrm{~A}$ & 119.8 & $\mathrm{C} 17-\mathrm{C} 16-\mathrm{H} 16 \mathrm{~A}$ & 119.6 \\
\hline $\mathrm{C} 4 \mathrm{~A}-\mathrm{C} 4-\mathrm{H} 4 \mathrm{~A}$ & 119.8 & $\mathrm{C} 18-\mathrm{C} 17-\mathrm{C} 16$ & $121.2(3)$ \\
\hline
\end{tabular}




\begin{tabular}{|c|c|}
\hline $\mathrm{C} 5-\mathrm{C} 4 \mathrm{~A}-\mathrm{C} 4$ & $123.6(3)$ \\
\hline $\mathrm{C} 5-\mathrm{C} 4 \mathrm{~A}-\mathrm{C} 8 \mathrm{~A}$ & $119.4(3)$ \\
\hline $\mathrm{C} 4-\mathrm{C} 4 \mathrm{~A}-\mathrm{C} 8 \mathrm{~A}$ & $117.0(3)$ \\
\hline $\mathrm{C} 6-\mathrm{C} 5-\mathrm{C} 4 \mathrm{~A}$ & $120.0(3)$ \\
\hline $\mathrm{C} 6-\mathrm{C} 5-\mathrm{H} 5 \mathrm{~A}$ & 120.0 \\
\hline $\mathrm{C} 4 \mathrm{~A}-\mathrm{C} 5-\mathrm{H} 5 \mathrm{~A}$ & 120.0 \\
\hline $\mathrm{C} 5-\mathrm{C} 6-\mathrm{C} 7$ & $121.1(3)$ \\
\hline $\mathrm{C} 5-\mathrm{C} 6-\mathrm{H} 6 \mathrm{~A}$ & 119.4 \\
\hline $\mathrm{C} 7-\mathrm{C} 6-\mathrm{H} 6 \mathrm{~A}$ & 119.4 \\
\hline $\mathrm{C} 8-\mathrm{C} 7-\mathrm{C} 6$ & $120.8(3)$ \\
\hline $\mathrm{C} 8-\mathrm{C} 7-\mathrm{H} 7 \mathrm{~A}$ & 119.6 \\
\hline $\mathrm{C} 6-\mathrm{C} 7-\mathrm{H} 7 \mathrm{~A}$ & 119.6 \\
\hline $\mathrm{C} 7-\mathrm{C} 8-\mathrm{C} 8 \mathrm{~A}$ & $119.2(2)$ \\
\hline $\mathrm{C} 7-\mathrm{C} 8-\mathrm{N} 9$ & $123.2(3)$ \\
\hline $\mathrm{C} 8 \mathrm{~A}-\mathrm{C} 8-\mathrm{N} 9$ & $117.6(2)$ \\
\hline $\mathrm{N} 1-\mathrm{C} 8 \mathrm{~A}-\mathrm{C} 8$ & $119.1(2)$ \\
\hline $\mathrm{N} 1-\mathrm{C} 8 \mathrm{~A}-\mathrm{C} 4 \mathrm{~A}$ & $121.6(2)$ \\
\hline $\mathrm{C} 8-\mathrm{C} 8 \mathrm{~A}-\mathrm{C} 4 \mathrm{~A}$ & $119.2(2)$ \\
\hline $\mathrm{C} 8 \mathrm{~A}-\mathrm{N} 1-\mathrm{C} 2-\mathrm{C} 3$ & $0.2(3)$ \\
\hline $\mathrm{Ag} 1-\mathrm{N} 1-\mathrm{C} 2-\mathrm{C} 3$ & $-161.74(19)$ \\
\hline $\mathrm{N} 1-\mathrm{C} 2-\mathrm{C} 3-\mathrm{C} 4$ & $3.0(4)$ \\
\hline $\mathrm{C} 2-\mathrm{C} 3-\mathrm{C} 4-\mathrm{C} 4 \mathrm{~A}$ & $-2.1(4)$ \\
\hline $\mathrm{C} 3-\mathrm{C} 4-\mathrm{C} 4 \mathrm{~A}-\mathrm{C} 5$ & $178.5(3)$ \\
\hline $\mathrm{C} 3-\mathrm{C} 4-\mathrm{C} 4 \mathrm{~A}-\mathrm{C} 8 \mathrm{~A}$ & $-1.9(3)$ \\
\hline $\mathrm{C} 4-\mathrm{C} 4 \mathrm{~A}-\mathrm{C} 5-\mathrm{C} 6$ & $178.6(3)$ \\
\hline $\mathrm{C} 8 \mathrm{~A}-\mathrm{C} 4 \mathrm{~A}-\mathrm{C} 5-\mathrm{C} 6$ & $-1.0(4)$ \\
\hline $\mathrm{C} 4 \mathrm{~A}-\mathrm{C} 5-\mathrm{C} 6-\mathrm{C} 7$ & $-2.1(5)$ \\
\hline $\mathrm{C} 5-\mathrm{C} 6-\mathrm{C} 7-\mathrm{C} 8$ & $2.0(5)$ \\
\hline $\mathrm{C} 6-\mathrm{C} 7-\mathrm{C} 8-\mathrm{C} 8 \mathrm{~A}$ & $1.3(4)$ \\
\hline $\mathrm{C} 6-\mathrm{C} 7-\mathrm{C} 8-\mathrm{N} 9$ & $-177.8(3)$ \\
\hline $\mathrm{C} 2-\mathrm{N} 1-\mathrm{C} 8 \mathrm{~A}-\mathrm{C} 8$ & $176.2(2)$ \\
\hline $\mathrm{Ag} 1-\mathrm{N} 1-\mathrm{C} 8 \mathrm{~A}-\mathrm{C} 8$ & $-19.6(2)$ \\
\hline $\mathrm{C} 2-\mathrm{N} 1-\mathrm{C} 8 \mathrm{~A}-\mathrm{C} 4 \mathrm{~A}$ & $-4.4(3)$ \\
\hline $\mathrm{Ag} 1-\mathrm{N} 1-\mathrm{C} 8 \mathrm{~A}-\mathrm{C} 4 \mathrm{~A}$ & $159.72(16)$ \\
\hline $\mathrm{C} 7-\mathrm{C} 8-\mathrm{C} 8 \mathrm{~A}-\mathrm{N} 1$ & $175.0(2)$ \\
\hline $\mathrm{N} 9-\mathrm{C} 8-\mathrm{C} 8 \mathrm{~A}-\mathrm{N} 1$ & $-5.8(3)$ \\
\hline $\mathrm{C} 7-\mathrm{C} 8-\mathrm{C} 8 \mathrm{~A}-\mathrm{C} 4 \mathrm{~A}$ & $-4.3(3)$ \\
\hline $\mathrm{N} 9-\mathrm{C} 8-\mathrm{C} 8 \mathrm{~A}-\mathrm{C} 4 \mathrm{~A}$ & $174.9(2)$ \\
\hline $\mathrm{C} 5-\mathrm{C} 4 \mathrm{~A}-\mathrm{C} 8 \mathrm{~A}-\mathrm{N} 1$ & $-175.2(2)$ \\
\hline $\mathrm{C} 4-\mathrm{C} 4 \mathrm{~A}-\mathrm{C} 8 \mathrm{~A}-\mathrm{N} 1$ & $5.2(3)$ \\
\hline $\mathrm{C} 5-\mathrm{C} 4 \mathrm{~A}-\mathrm{C} 8 \mathrm{~A}-\mathrm{C} 8$ & $4.2(3)$ \\
\hline $\mathrm{C} 4-\mathrm{C} 4 \mathrm{~A}-\mathrm{C} 8 \mathrm{~A}-\mathrm{C} 8$ & $-175.4(2)$ \\
\hline $\mathrm{C} 7-\mathrm{C} 8-\mathrm{N} 9-\mathrm{Ag} 1$ & $-153.5(2)$ \\
\hline $\mathrm{C} 8 \mathrm{~A}-\mathrm{C} 8-\mathrm{N} 9-\mathrm{Ag} 1$ & $27.4(2)$ \\
\hline
\end{tabular}

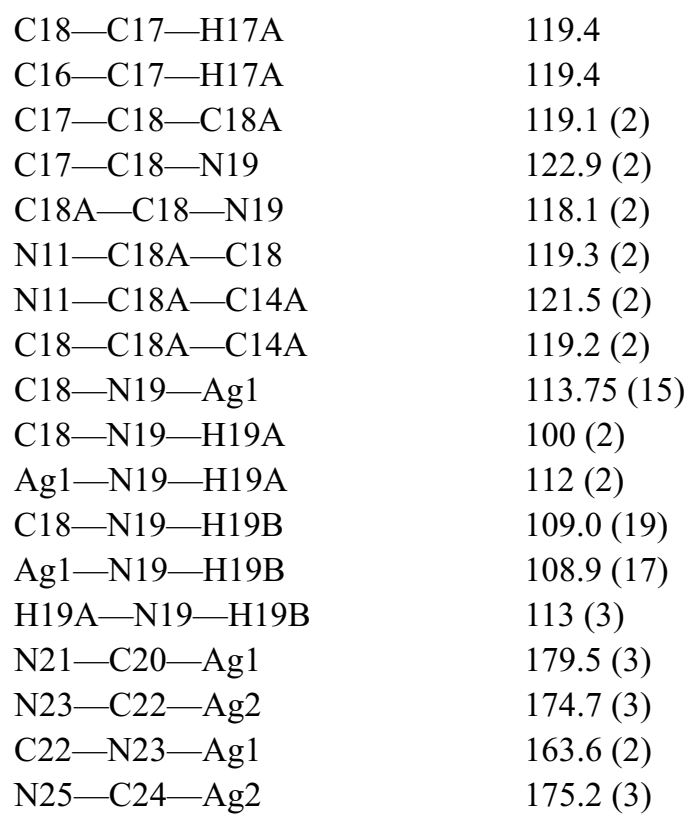


Hydrogen-bond geometry $\left(\AA,{ }^{\circ}\right)$

\begin{tabular}{lllll}
\hline$D-\mathrm{H} \cdots A$ & $D-\mathrm{H}$ & $\mathrm{H} \cdots A$ & $D \cdots A$ & $D-\mathrm{H} \cdots A$ \\
\hline $\mathrm{N} 9-\mathrm{H} 9 A^{\cdots} \cdots \mathrm{N} 21^{\mathrm{i}}$ & $0.79(3)$ & $2.36(3)$ & $3.143(4)$ & $169(3)$ \\
$\mathrm{N} 9-\mathrm{H} 9 B \cdots \mathrm{N} 21^{\mathrm{ii}}$ & $0.85(3)$ & $2.23(3)$ & $3.075(3)$ & $172(3)$ \\
$\mathrm{N} 19-\mathrm{H} 19 A \cdots \mathrm{N} 21^{\mathrm{ii}}$ & $0.77(3)$ & $2.48(3)$ & $3.205(4)$ & $157(3)$ \\
$\mathrm{N} 19-\mathrm{H} 19 B \cdots \mathrm{N} 25^{\mathrm{iii}}$ & $0.90(3)$ & $2.19(3)$ & $3.087(4)$ & $175(3)$
\end{tabular}

Symmetry codes: (i) $-x+1,-y+2,-z+1$; (ii) $x, y-1, z$; (iii) $-x+2, y-1 / 2,-z+1 / 2$. 